\title{
Theory of electron spin relaxation in $\mathrm{ZnO}$
}

\author{
N. J. Harmon, ${ }^{1}$ W. O. Putikka, ${ }^{1}$ and R. Joynt ${ }^{2}$ \\ ${ }^{1}$ Department of Physics, Ohio State University, 191 W. Woodruff Avenue, Columbus, Ohio 43210, USA \\ ${ }^{2}$ Department of Physics, University of Wisconsin-Madison, 1150 University Avenue, Madison, Wisconsin 53705, USA
}

(Received 21 August 2008; revised manuscript received 13 February 2009; published 11 March 2009)

\begin{abstract}
Doped $\mathrm{ZnO}$ is a promising material for spintronics applications. For such applications, it is important to understand the spin dynamics and particularly the spin relaxation times of this II-VI semiconductor. The spin relaxation time $\tau_{s}$ has been measured by optical orientation experiments, and it shows a surprising nonmonotonic behavior with temperature. We explain this behavior by invoking spin exchange between localized and extended states. Interestingly, the effects of spin-orbit coupling are by no means negligible, in spite of the relatively small valence-band splitting. This is due to the wurtzite crystal structure of $\mathrm{ZnO}$. Detailed analysis allows us to characterize the impurity binding energies and densities, showing that optical orientation experiments can be used as a characterization tool for semiconductor samples.
\end{abstract}

DOI: 10.1103/PhysRevB.79.115204

PACS number(s): 72.25.Rb, 71.55.Gs

\section{INTRODUCTION}

Zinc oxide has been the subject of considerable experimental and theoretical investigation for many years. ${ }^{1}$ Its band gap is in the near ultraviolet, making it useful as a transparent conductor and as sunscreen. Its piezoelectricity opens up transduction applications. The activity has intensified more recently because of the possibility that $\mathrm{ZnO}$ might be useful for spintronics or spin-based quantum computation. It has been predicted to be a room-temperature ferromagnet when doped with $\mathrm{Mn}^{2}$ Furthermore, its spin-orbit coupling is generally thought to be very weak compared with GaAs. The usual measure of the strength of spin-orbit coupling in semiconductors is the energy splitting at the top of the valence band. It is said that the spin-orbit coupling is negligible in $\mathrm{ZnO}$ because the valence-band splitting is $-3.5 \mathrm{meV},{ }^{3}$ as opposed to $340 \mathrm{meV}$ for GaAs. Smaller spin-orbit coupling should lead to long spin relaxation times. Long relaxation times are required if spin information is to be transported over appreciable distances.

The spin relaxation time $\tau_{s}$ has been measured by Ghosh et al. ${ }^{4}$ to be about $20 \mathrm{~ns}$ from 0 to $20 \mathrm{~K}$ in optical orientation experiments. $\tau_{s}$ is sometimes called $T_{2}^{*}$ even in the absence of an external field. Since the data from Ref. 4 used in this paper were taken at zero field, the relaxation time is taken to be $\tau_{s}$ to avoid confusion with experiments conducted at finite field. The data show two surprising features. First, the relaxation times are actually somewhat shorter than the longest relaxation times in GaAs, which are about 100 ns. $^{5}$ One might expect the opposite given the relative strength of spinorbit coupling in the two materials. Second, $\tau_{s}$ shows a nonmonotonic temperature dependence, first increasing slightly and then rapidly decreasing - but increasing temperature usually promotes spin relaxation.

In this paper, we show that a theory previously developed for $\tau_{s}$ in GaAs (Ref. 6) can account for these observations. The theory must be modified to take account of the different impurity levels and binding energies of $\mathrm{ZnO}$. This is important, because, in spite of intensive investigation, the nature of the impurities that govern the electrical properties of $\mathrm{ZnO}$ remains controversial, and our analysis sheds some light on this issue. Even more interestingly, it turns out that the wurtzite crystal structure has very important consequences for the D'yakonov-Perel' (DP) (Ref. 7) scattering that dominates the relaxation at higher temperatures. Thus the crystal structure must be taken into account fully. The final message will be that the "weak" spin-orbit coupling of $\mathrm{ZnO}$ is not negligible for spin relaxation, and it does not lead to long relaxation times.

In Sec. II we give the background information for $\mathrm{ZnO}$. Section III is devoted to a derivation of the equations of motion for the spins. In Sec. IV the computational method is described. The determination of the parameters in the equations of motion is a separate task. The most important of the parameters is that which controls the DP spin relaxation. Since the calculation of these parameters is not straightforward, we devote Sec. V to that. Section VI gives the results. Section VII is the conclusions and puts the results into context.

\section{BACKGROUND}

In $\mathrm{ZnO}$ produced by the hydrothermal method, it is generally thought that there are two sets of impurity states, one shallow and quasihydrogenic, one deep and very well localized. ${ }^{8,9}$ Their precise physical nature is not known. In the case of the deep impurity, it is believed that a lattice defect accompanies the chemical impurity. The binding energies are in the range of a few $10 \mathrm{~s}$ of $\mathrm{meV}$ for the shallow impurity and a few $100 \mathrm{~s}$ of meV for the deep impurity. We shall demonstrate below that the optical orientation data can put bounds on these numbers.

$\mathrm{ZnO}$ crystallizes in the wurtzite structure rather than the zinc-blende structure familiar from the III-V compounds. This has very important implications for the conduction-band states. The spin-orbit interaction lifts the spin degeneracy in the conduction band. In zinc-blende structures crystal symmetry implies that the splitting is cubic in the magnitude of the wave vector $k$, but in the wurtzite structure the splitting is linear. ${ }^{10}$ However, the spin relaxation time of the low-lying conduction-band states depends mainly on the spin splitting 
near the conduction-band minimum, and this is larger in $\mathrm{ZnO}$ than in GaAs for small enough $k$.

In optical orientation experiments, electrons are excited from the valence band to the conduction band by circularly polarized light tuned close to the band-gap energy (pump step). The population of conduction electrons so created is spin-polarized. ${ }^{11}$ Energy relaxation then occurs on a short time scale ( $\leq 1 \mathrm{~ns}$ ), but most of this relaxation is from spinconserving processes, so there is a longer time scale (or time scales) on which the spin of the system relaxes. When an external magnetic field is present, the time scale to relax the transverse component of the net magnetization is called $\tau_{s}$. It is measured using Faraday rotation or the Kerr effect (probe step).

The important physical point is that the fast energy relaxation leads to a thermal charge distribution for the electrons by the time $1 \mathrm{~ns}$ has elapsed, but the spin distribution relaxes on longer time scales. The thermal charge distribution means that the localized donor impurity states are mostly full at the relatively low temperatures of the experiment. The spins of the localized electrons must be included along with the conduction electron spins. The spins of localized and extended states can be interchanged by the exchange coupling, a process we call cross-relaxation. This is often a rather fast process and is particularly important when the relaxation times of the localized and extended states are very different in magnitude. In GaAs this process is important in all the regimes of temperature, applied field, and impurity density that have been studied, and it is important in $\mathrm{ZnO}$ as well. In Sec. III we derive a set of modified Bloch equations to describe the aforementioned spin dynamics.

\section{MODIFIED BLOCH EQUATIONS}

We consider a conduction electron in the semiclassical approximation. It moves as a wave packet with a welldefined momentum and scatters from impurities and phonons at time intervals of average length $\tau_{p}$, where $\tau_{p}$ is the momentum relaxation time. Its spin operator is $\mathbf{s}_{c}$. The spindependent part of its Hamiltonian in the absence of an external magnetic field is

$$
H^{c}=H_{1}^{c}+H_{2}^{c}=-\frac{1}{2 \hbar} \sum_{i} J\left(\mathbf{r}-\mathbf{R}_{i}\right) \mathbf{s}_{i} \cdot \mathbf{s}_{c}-g \frac{\mu_{B}}{\hbar} \mathbf{b}(t) \cdot \mathbf{s}_{c} .
$$

The first term, $H_{1}^{c}$, is the exchange interaction with impurity spins $\mathbf{s}_{i}$ located at positions $\mathbf{R}_{i}$. It is the same interaction that is responsible for the Kondo effect, but the temperatures here are all much greater than the Kondo temperature. The range of the function $J\left(\mathbf{r}-\mathbf{R}_{i}\right)$ is roughly $a_{B}$, where $a_{B}$ is the effective Bohr radius. The second term, $H_{2}^{c}$, represents other spin relaxation mechanisms that we model as a small random classical field $\mathbf{b}(t)$ with a correlation time much shorter than $\tau_{s}$. An analogous Hamiltonian $H^{l}$ can be written for a localized electron.

First, we concentrate on the spin dynamics resulting from the spin-spin term and ignore the second term. In the dilute limit $\left(a_{B} n_{\mathrm{imp}}^{1 / 3} \ll 1\right)$, a conduction electron encounters impuri- ties with randomly aligned spins if no short-range order is present in the impurity system. An effective field from the impurity spin affects the conduction electron when it is within $\sim a_{B}$ of the impurity. When $\left|\mathbf{r}-\mathbf{R}_{i}\right|>a_{B}$, the conduction electron proceeds unhindered by the effective field. This effective field is a result of the exchange potential. An itinerant electron will spend an average time of $a_{B} / v$ within the range of the effective field where $v$ is the velocity of the electron. Thus the time between encounters ${ }^{12}$ is $1 / n_{l} a_{B}^{2} v$.

In a semiclassical picture the spin of the itinerant electron undergoes precession of magnitude $\Delta \phi=J a_{B} / 2 v$ through a random angle during each encounter with an impurity. The spin of the impurity electron also precesses but with angle $-\Delta \phi$. Since the sum of spins, $\mathbf{s}_{c}+\mathbf{s}_{l}$, commutes with $H_{1}^{c}$ $+H_{1}^{l}$, the total spin in the system must be conserved. However the spin in each subsystem may shift between one another; this is cross-relaxation.

It turns out for the parameters of the system under consideration that $\Delta \phi \sim 1$, and we then find that

$$
\tau_{c}^{\mathrm{cr}} \sim \frac{1}{n_{l} a_{B}^{2} v}
$$

which implies that the spin is essentially randomized after one impurity encounter.

If we consider an ensemble of conduction electrons with a net magnetization $m_{c}$, this magnetization is exchanged at a rate of $1 / \tau_{c}^{\mathrm{cr}}$. As previously mentioned, any magnetization lost from the conduction electrons must be gained by the localized electrons and vice versa. For clarity we write $1 / \tau_{c}^{\mathrm{cr}}=n_{l} / \gamma^{\mathrm{cr}}$ and $1 / \tau_{l}^{\mathrm{cr}}=n_{c} / \gamma^{\mathrm{cr}}$ where $\gamma^{\mathrm{cr}}=1 / a_{B}^{2} v$.

We now examine the second term of the Hamiltonian

$$
H_{2}^{c}(t)=-\frac{1}{2} g \mu_{B}\left[b_{x}(t) \sigma_{x}+b_{y}(t) \sigma_{y}+b_{z}(t) \sigma_{z}\right] .
$$

This Hamiltonian relaxes the conduction electron spin. To extract a relaxation rate from this Hamiltonian, we use the equation of motion

$$
\frac{d \rho(t)}{d t}=\frac{i}{\hbar}\left[\rho(t), H_{2}^{c}(t)\right],
$$

where $\rho(t)$ is the $2 \times 2$ spin density matrix for an electron of a given momentum. We assume that the total density matrix for the conduction electron factorizes; we neglect offdiagonal terms that come from correlations. By iteration, we can write this equation as

$$
\begin{aligned}
\left\langle\frac{d \rho(t)}{d t}\right\rangle= & \frac{i}{\hbar}\left\langle\left[\rho(0), H_{2}^{c}\left(t^{\prime}\right)\right]\right\rangle \\
& -\frac{1}{\hbar^{2}} \int_{0}^{t}\left\langle\left[\left[\rho\left(t^{\prime}\right), H_{2}^{c}\left(t^{\prime}\right)\right], H_{2}^{c}(t)\right]\right\rangle d t^{\prime},
\end{aligned}
$$

where the angular brackets indicate averaging over all orientations of $b(t)$. To simplify notation, from now on angular brackets will be suppressed on the density matrix. Since $\left\langle b_{i}(t)\right\rangle=0$, the first term is zero. We assume that different directions of $b_{i}$ are uncorrelated and (since the external field is zero) different direction are equivalent. Then we have $\left\langle b_{i}(t) b_{j}\left(t^{\prime}\right)\right\rangle=\left\langle b(t) b\left(t^{\prime}\right)\right\rangle \delta_{i, j}$. Therefore, Eq. (5) reduces to 


$$
\frac{d \rho(t)}{d t}=-\frac{g^{2} \mu_{B}^{2}}{2 \hbar^{2}} \int_{0}^{t} \sum_{i}\left[\rho\left(t^{\prime}\right), \sigma_{i}\right] \sigma_{i}\left\langle b(t) b\left(t^{\prime}\right)\right\rangle d t^{\prime} .
$$

The correlation function is assumed to be stationary in time so $\left\langle b(t) b\left(t^{\prime}\right)\right\rangle=g\left(t^{\prime}-t\right)=g(\tau) \cdot{ }^{13}$ If the correlation time of the $b$ fluctuations, $\tau_{e}$, is short, $\rho$ will not change on that timescale and $g(\tau)$ will be nearly a $\delta$ function (Markov approximation). Equation (6) can then be written as

$$
\frac{d \rho(t)}{d t}=-\frac{2 g^{2} \mu_{B}^{2}}{\hbar^{2}} \frac{1}{4} \sum_{i}\left[\rho(t), \sigma_{i}\right] \sigma_{i} \int_{0}^{\infty}\left\langle b(t) b\left(t^{\prime}\right)\right\rangle d t^{\prime} .
$$

The integral is approximated by $\left\langle b^{2}\right\rangle \tau_{e}$. Define the relaxation time scale $\tau_{c}$ by

$$
\frac{1}{\tau_{c}}=2\left(\frac{g \mu_{B}}{\hbar}\right)^{2}\left\langle b^{2}\right\rangle \tau_{e}
$$

giving

$$
\frac{d \rho(t)}{d t}=-\frac{1}{4 \tau_{c}} \sum_{i}\left[\rho(t), \sigma_{i}\right] \sigma_{i} .
$$

The density matrix can be expanded in Pauli spin matrices

$$
\rho(t)=\frac{1}{2} I+\frac{1}{2} \sum_{i} m_{i}(t) \sigma_{i},
$$

where $I$ is the $2 \times 2$ identity matrix and $m_{i}=\operatorname{Tr}\left(\sigma_{i} \rho\right)$ is the expected value of the magnetization. Inserting Eq. (10) in Eq. (9) and matching coefficients of Pauli matrices gives a set of equations for the dynamics of $\mathbf{m}$. For instance for conduction electron magnetization $m_{c}$ in the $x$ direction, $d m_{c} / d t=\operatorname{Tr}\left(\sigma_{x} d \rho / d t\right)=-m_{c} / \tau_{c}$. As with $H_{1}^{c}$, similar expressions for the localized magnetization $m_{l}$ can be found: $d m_{l} / d t=\operatorname{Tr}\left(\sigma_{x} d \rho / d t\right)=-m_{l} / \tau_{l}$.

By combining the effects of $H_{1}=H_{1}^{c}+H_{1}^{l}$ and $H_{2}=H_{2}^{c}$ $+H_{2}^{l}$, the modified Bloch equations for the magnetizations can be expressed as

$$
\begin{gathered}
\frac{d m_{c}}{d t}=-\left(\frac{1}{\tau_{c}}+\frac{n_{l}}{\gamma^{\mathrm{cr}}}\right) m_{c}+\frac{n_{c}}{\gamma^{\mathrm{cr}}} m_{l}, \\
\frac{d m_{l}}{d t}=\frac{n_{l}}{\gamma^{\mathrm{cr}}} m_{c}-\left(\frac{1}{\tau_{l}}+\frac{n_{c}}{\gamma^{\mathrm{cr}}}\right) m_{l}
\end{gathered}
$$

for two spin systems - itinerant and localized spins. $\tau_{c}$ and $\tau_{l}$ in Eq. (11) are now described in terms of well known relaxation mechanisms which will be discussed in Sec. IV. This model was successfully applied to GaAs. ${ }^{6}$ For $\mathrm{ZnO}$, these Bloch equations are easily extended to account for the multiple-type impurities present.

\section{METHOD}

We now seek to write equations like those of Eq. (11) with regard given to the two types of impurities in $\mathrm{ZnO}-$ shallow and deep. As mentioned above, we find that the cross-relaxation is important to understand the data. These rates come from the Kondo-type $J \mathbf{s}_{l} \cdot \mathbf{s}_{c}$ interaction between an impurity spin $\mathbf{s}_{l}$ and a conduction-band spin $\mathbf{s}_{c}$. An expression for $J$ in terms of tight-binding parameters can be derived using the Schrieffer-Wolf transformation. ${ }^{14}$ One expects that the cross-relaxation between conduction and shallow donor electrons to be much more rapid than the cross-relaxation between conduction and deep donor electrons because of the greater binding energy of the deep impurity and its larger on-site Coulomb energy. This is confirmed by the fit to the data. In fact we find that terms involving cross-relaxation between the deep donors and either the conduction-band electrons or the shallow donor electrons can be neglected. With these simplifications, for $\mathrm{ZnO}$ Eq. (11) extends to

$$
\begin{gathered}
\frac{d m_{c}}{d t}=-\left(\frac{1}{\tau_{c}}+\frac{n_{l s}}{\gamma_{c, s}^{\mathrm{cr}}}\right) m_{c}+\frac{n_{c}}{\gamma_{c, s}^{\mathrm{cr}}} m_{l s}, \\
\frac{d m_{l s}}{d t}=\frac{n_{l s}}{\gamma_{c, s}^{\mathrm{cr}}} m_{c}-\left(\frac{1}{\tau_{l s}}+\frac{n_{c}}{\gamma_{c, s}^{\mathrm{cr}}}\right) m_{l s}, \\
\frac{d m_{l d}}{d t}=-\frac{1}{\tau_{l d}} m_{l d} .
\end{gathered}
$$

In this equation, $m_{c}, m_{l s}$, and $m_{l d}$ stand for the magnetizations of the conduction electrons, the electrons on shallow impurities, and the electrons on deep impurities, respectively. The $n$ 's denote the corresponding volume densities. Each of the populations has a relaxation time $\tau_{c}, \tau_{l s}$, and $\tau_{l d}$. From Eq. (12), we can then find the magnetization as a function of time.

Standard methods can be used to solve these differential equations. The solutions yield a time dependence of the total magnetization, $m(t)=m_{c}(t)+m_{l s}(t)+m_{l d}(t)$, to be a sum of three exponentials, $\exp \left(-\Gamma_{+} t\right), \exp \left(-\Gamma_{-} t\right)$, and $\exp \left(-\Gamma_{d} t\right)$ where

$$
\Gamma_{ \pm}=\frac{1}{2}\left(\frac{1}{\tau_{c}}+\frac{1}{\tau_{l s}}+\frac{n_{c}+n_{l s}}{\gamma_{c, s}^{\mathrm{cr}}} \pm S\right), \quad \Gamma_{d}=\frac{1}{\tau_{l d}}
$$

with $S$ given by

$$
S=\sqrt{\left(\frac{1}{\tau_{l s}}-\frac{1}{\tau_{c}}+\frac{n_{c}-n_{l s}}{\gamma_{c, s}^{\mathrm{cr}}}\right)^{2}+\frac{4 n_{c} n_{l s}}{\gamma_{c, s}^{\mathrm{cr} 2}} .}
$$

No net moment can exist on the deep donor sites since no moment is excited into the deep states on account of them being significantly below the conduction band, and no net moment cross relaxes into these states. Therefore $\Gamma_{d}$ can be ruled out as being the observed relaxation rate. In the regime that $\left(n_{l s}+n_{c}\right) / \gamma_{c, s}^{\mathrm{cr}} \gg 1 / \tau_{c}, 1 / \tau_{l s}$, the rate $\Gamma_{+}$simplifies to $\left(n_{c}+n_{l s}\right) / \gamma_{c, s}^{\mathrm{cr}}$ and is very rapid and the rate $\Gamma_{-}$is slower,

$$
\Gamma_{-}=\frac{n_{c}}{n_{c}+n_{l s}} \frac{1}{\tau_{c}}+\frac{n_{l s}}{n_{c}+n_{l s}} \frac{1}{\tau_{l s}} .
$$

We fit the data with this equation and associate it with $\tau_{s}$. We see that the relaxation rate depends on two factors: the thermodynamic occupations of the shallow donors (the deep donors are always nearly full in the temperature range studied here) and form of the relaxation rates for the conduction and localized shallow states. 
The densities can be computed using standard formulas from equilibrium statistical mechanics, since we deal only with time scales long compared to the fast energy relaxation scale. As a function of temperature $T$, the ratio $n_{c} / n_{l s}$ naturally increases rapidly as $T \rightarrow\left|\varepsilon_{l s}\right| / k_{B}$, where $\varepsilon_{l s}$ is the binding energy of the shallow impurity. $\left|\varepsilon_{l d}\right|$ is so large that these states are always occupied at the experimental temperatures, which range from 5 to $80 \mathrm{~K}$.

$\tau_{c}$ is fairly complicated to calculate because there are several mechanisms that can relax the conduction electron spins. The simplest such mechanism is the Elliot-Yafet (EY) process ${ }^{15}$ that arises from spin mixing in the wave functions. When a conduction electron is scattered by a spinindependent potential from state $\mathbf{k}$ to state $\mathbf{k}^{\prime}$, the initial and final states are not eigenstates of the spin projection operator $S_{z}$ so the process relaxes the spin. The rate of relaxation due to the EY process is well known to be of the form $1 / \tau_{\mathrm{EY}}$ $=\alpha_{\mathrm{EY}} T^{2} / \tau_{p}(T)$ where $\alpha_{\mathrm{EY}}$ is a material-dependent parameter and $\tau_{p}$ is the momentum relaxation time. ${ }^{16} \mathrm{We}$ estimate $\alpha_{\mathrm{EY}}(t h)=4.6 \times 10^{-15} \mathrm{~K}^{-2}$. The Bir-Aronov-Pikus mechanism ${ }^{17}$ arises from the scattering of electron and holes. This relaxation mechanism is commonly considered to be negligible in $n$-type materials like those under consideration here since the number of holes is small. ${ }^{18}$ The DP mechanism ${ }^{7}$ arises from the ordinary scattering of conduction-band states. Since this has not previously been calculated in a wurtzite structure, we devote Sec. V to it. This calculation yields an expression for $\tau_{c}$ as a function of temperature.

$\tau_{l s}$ and $\tau_{l d}$ are due to non-spin-conserving anisotropic exchange Dzyaloshinsky-Moriya (DM) interactions. ${ }^{19,20}$ The anisotropic exchange term is important. It arises from spinorbit coupling and produces a term proportional to $\mathbf{d} \cdot \mathbf{s}_{\mathbf{1}}$ $\times \mathbf{s}_{\mathbf{2}}$ where $\mathbf{d}$ is related to the interspin separation and the exchange integral between the wave function on sites 1 and 2. However, it is not possible to calculate it in detail when the nature of the impurities is not well known. We estimate the rate as $1 / \tau_{\mathrm{DM}}=\alpha_{\mathrm{DM}}\left(n_{\mathrm{imp}, s}+n_{\mathrm{imp}, d}\right)$ where $n_{\mathrm{imp}, s}$ and $n_{\mathrm{imp}, d}$ are the total impurity concentrations of the shallow and deep impurity, respectively, and $\alpha_{\mathrm{DM}}$ has a weak temperature dependence that we neglect. The main contribution comes from the overlap of the shallow impurity wave functions, which we take to be hydrogenic, with the deep impurity wave functions, which we take to be well-localized on an atomic scale. The details of how to estimate the resulting relaxation may be found in Refs. 6, 21, and 22. The numerical value we find from theory is $\alpha_{\mathrm{DM}}(t h)=1.12 \times 10^{-20} \mathrm{~cm}^{3} \mathrm{~ns}^{-1}$. When nuclei possess nonzero magnetic moments, the hyperfine interaction between electron and nuclear spin is a source of spin relaxation for localized electrons. ${ }^{23}$ However, zero nuclear spin isotopes of $\mathrm{Zn}$ and $\mathrm{O}$ are $96 \%$ and $99.5 \%$ naturally abundant, respectively. Therefore we rule out the hyperfine interaction from being an observed relaxation mechanism in Ref. 4.

\section{DP MECHANISM IN WURTZITE CRYSTAL STRUCTURES}

The conduction-band states undergo ordinary impurity and phonon scattering. Each scattering event give a change in the wave vector $\mathbf{k}$, which in turn changes the effective magnetic field on the spin that comes from spin-orbit coupling. This fluctuating field relaxes the spin. The effective field strength is proportional to the conduction-band spin splitting. Bulk zinc-blende crystals have conduction-band splittings cubic-in- $k$ due to bulk inversion asymmetry (Dresselhaus effect). ${ }^{24}$ In addition to cubic terms, bulk wurtzite conduction bands also possess spin splittings proportional to linear terms in $k$ due to the hexagonal $c$ axis which gives bulk wurtzite a reflection asymmetry similar to the Rashba effect. ${ }^{10,25-27}$ We can write the spin-orbit Hamiltonian to include both the Rashba and Dresselhaus terms:

$$
H_{\mathrm{so}}(\mathbf{k})=\left[\alpha \boldsymbol{\kappa}_{1}+\gamma \boldsymbol{\kappa}_{3}\right] \cdot \boldsymbol{\sigma},
$$

where $\kappa_{1}=\left(k_{y},-k_{x}, 0\right)$ is linear-in- $k, \kappa_{3}=\left(k_{\|}^{2}-b k_{z}^{2}\right)\left(k_{y},-k_{x}, 0\right)$ is cubic-in- $k, \boldsymbol{\sigma}=\left(\sigma_{x}, \sigma_{y}, \sigma_{z}\right)$ are the Pauli spin matrices, and $\alpha, \gamma$ are spin splitting coefficients. ${ }^{10,26,28}$ The parameter $b$ is roughly equal to four for all wurtzite materials. ${ }^{28}$ Note that there is no spin splitting along the hexagonal axis $(z)$.

The linear-in- $k$ term dominates and we can determine the spin relaxation rate by following the treatment given by Pikus and Titkov in Ref. 29 which yields the following relaxation rates:

$$
\begin{gathered}
\frac{1}{\tau_{\mathrm{DP}, i i}^{(1)}}=\widetilde{\tau}_{p} \frac{4 \alpha^{2}}{\hbar^{2}}\left({\overline{\kappa_{1}}}^{2}-{\overline{\kappa_{1, i}}}^{2}\right), \\
\frac{1}{\tau_{\mathrm{DP}, i \neq j}^{(1)}}=\widetilde{\tau}_{p} \frac{4 \alpha^{2}}{\hbar^{2}} \overline{\kappa_{1, i} \kappa_{1, j}},
\end{gathered}
$$

where the overbar denotes angular averaging, and $i, j$ denote the Cartesian components of $\boldsymbol{\kappa}_{1}$. The momentum relaxation rate is defined as

$$
\frac{1}{\widetilde{\tau}_{p}}=\int_{-1}^{1} \sigma(\theta)(1-\cos \theta) d \cos \theta,
$$

where $\sigma(\theta)$ is the scattering cross section and $\theta$ is the angle between initial and final k. ${ }^{29}$ In bulk wurtzite $\overline{\kappa_{1, \|}}{ }^{2}={\overline{\kappa_{1, x}}}_{2}$ $={\overline{\kappa_{1, y}}}^{2}=k^{2} / 3,{\overline{\kappa_{1, z}}}^{2}=0$, and in the unstrained crystal, $\overline{\kappa_{1, i} \kappa_{1, j}}$ $=0$ for $i \neq j$. From Eq. (17), we can write

$$
\frac{1}{\tau_{\mathrm{DP}, \|}^{(1)}}=\frac{2}{\tau_{\mathrm{DP}, z}^{(1)}}=\frac{4}{3} \frac{\alpha^{2}}{\hbar^{2}} \widetilde{\tau}_{p} k^{2}=\frac{8}{3} \frac{m^{*} \alpha^{2}}{\hbar^{4}} \widetilde{\tau}_{p} E_{\mathbf{k}},
$$

where $m^{*}$ is the electron effective mass and $E_{\mathbf{k}}$ is the energy $\hbar^{2} k^{2} / 2 m^{*}$. This result can be Boltzmann averaged (denoted by angle brackets) to obtain

$$
\frac{1}{\tau_{\mathrm{DP}}^{(1)}(T)}=\left\langle\frac{1}{\tau_{\mathrm{DP}, \|}^{(1)}}\right\rangle=\frac{8}{3} \frac{m^{*} \alpha^{2}}{\hbar^{4}}\left\langle\widetilde{\tau}_{p} E_{\mathbf{k}}\right\rangle=\alpha_{\mathrm{DP}}^{(1)} \tau_{p}(T) T,
$$

where $\quad \alpha_{\mathrm{DP}}^{(1)}=4 m^{*} \alpha^{2} k_{B} / \hbar^{4} \quad$ and $\quad \tau_{p}(T)=\left\langle\tilde{\tau}_{p} E_{\mathbf{k}}\right\rangle /\left\langle E_{\mathbf{k}}\right\rangle$ $=2\left\langle\widetilde{\tau}_{p} E_{\mathbf{k}}\right\rangle / 3 k_{B} T$. The temperature-dependent momentum relaxation time, $\tau_{p}(T)$, can be determined from electron mobility $\left(\mu_{e}\right)$ measurements from $\mu_{e}=e \tau_{p}(T) / m^{*}$, where $e$ is the charge of an electron. $\alpha$ has been calculated ${ }^{10}$ to be 1.1 $\times 10^{-4} \mathrm{eV} \mathrm{nm}$ which gives a theoretical value of $\alpha_{\mathrm{DP}}^{(1)}(t h)$ $=34.6 \mathrm{~K}^{-1} \mathrm{~ns}^{-2}$.

Similarly, the cubic-in- $k$ term can be calculated to be 


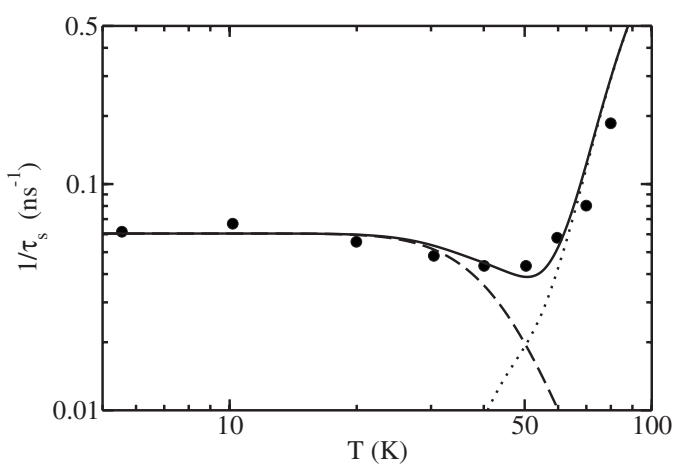

FIG. 1. Plot of $1 / \tau_{s}$ vs temperature. Points are experiment of Ref. 4. Dashed curve: $\left[n_{l s} /\left(n_{c}+n_{l s}\right)\right]\left(1 / \tau_{\mathrm{DM}}\right)$. Dotted curve: $\left[n_{c} /\left(n_{c}+n_{l s}\right)\right]\left(1 / \tau_{\mathrm{DP}}\right)$. Solid curve: total $1 / \tau_{s} . \quad n_{\mathrm{imp}, s}=6.0$ $\times 10^{14} \mathrm{~cm}^{-3}, \quad n_{\text {imp }, d}=5.0 \times 10^{17} \mathrm{~cm}^{-3}, \quad \varepsilon_{l s}=-23 \mathrm{meV}, \quad$ and $\varepsilon_{l d}=-360 \mathrm{meV}$.

$$
\frac{1}{\tau_{\mathrm{DP}}^{(3)}(T)}=\frac{1}{\tau_{\mathrm{DP}, \|}^{(3)}(T)}=\frac{2}{\tau_{\mathrm{DP}, z}^{(3)}(T)}=\alpha_{\mathrm{DP}}^{(3)} \tau_{p}(T) T^{3},
$$

where $\alpha_{\mathrm{DP}}^{(3)}=80 Q \gamma^{2} m^{* 3} k_{B}^{3} / 3 \hbar^{8}$ where the dimensionless quantity $Q$ depends on the type of scattering and is of order unity. $\gamma$ has been calculated ${ }^{10}$ to be $3.3 \times 10^{-4} \mathrm{eV} \mathrm{nm}^{3}$ which yields $\alpha_{\mathrm{DP}}^{(3)}(t h)=2.0 \times 10^{-4} \mathrm{~K}^{-3} \mathrm{~ns}^{-2}$. The sample from which the momentum relaxation times $\tau_{p}(T)$ were extracted ${ }^{30}$ was hydrothermally grown by the same company as Ghosh et al. sample in Ref. 4.

\section{RESULTS AND DISCUSSION}

In Fig. 1 we show that temperature dependence of $\tau_{s}$ as measured in a bulk $\mathrm{ZnO}$ sample and our fit [using Eq. (15)] to the data. It is seen immediately that the temperature dependence is not monotonic and that this is well reproduced by the theory. The reason is simple. At low temperatures $T$ $\ll\left|\varepsilon_{l s}\right| / k_{B}$ nearly all the electrons are in localized states. These states relax by the temperature-independent DM mechanism: $1 / \tau_{l s}=1 / \tau_{\mathrm{DM}}$. This mechanism alone determines the $T=0$ values. When $T$ approaches $\left|\varepsilon_{l s}\right| / k_{B}$, the deep impurities are all occupied but the rest of the population is shared by shallow localized and conduction-band states. Initially, the conduction-band electrons have a longer spin lifetime because impurity scattering is frequent at low temperatures so the DP mechanism that relaxes them is not very effective. However, the DP mechanism increases rapidly as $T$ increases and the $\tau_{s}$ curve turns around. At $T \gg\left|\varepsilon_{l s}\right| / k_{B}$, the shallow impurity level is empty and the relaxation is dominated by the DP mechanism in the conduction band: $1 / \tau_{c}=1 / \tau_{\mathrm{DP}}^{(1)}(T)$.

At this point it is necessary to point out why only the linear-in- $T$ DP mechanism is needed to explain the observed conduction spin relaxation. The other two viable candidates (cubic DP and EY) for relaxation are much too weak to explain the observed relaxation times in $\mathrm{ZnO}$. We use the calculated values for $\alpha_{\mathrm{DP}}^{(1)}(t h)$ and $\alpha_{\mathrm{DP}}^{(3)}(t h)$ in the previous section to obtain the relative relaxation efficiencies between the linear and cubic DP mechanism terms:

$$
\frac{1 / \tau_{\mathrm{DP}}^{(1)}}{1 / \tau_{\mathrm{DP}}^{(3)}}=\frac{\alpha_{\mathrm{DP}}^{(1)}(t h)}{\alpha_{\mathrm{DP}}^{(3)}(t h) T^{2}}=\frac{1.73 \times 10^{5} \mathrm{~K}^{2}}{T^{2}},
$$

which demonstrates that the efficiency of the cubic-in- $T$ term does not become comparable to the linear-in- $T$ term at temperatures below $416 \mathrm{~K}$ which is far above the temperature range investigated here. In fact the cubic-in- $T$ term does not even reach one-tenth the efficiency of the linear-in- $T$ term in the temperature range investigated here. For this reason we can confidently ignore the cubic-in- $T$ DP mechanism term in our fit. The crystal structure of $\mathrm{ZnO}$ therefore makes its spin relaxation qualitatively different from spin relaxation in bulk $n$-GaAs. We also compare the efficiencies of the DP and EY mechanisms:

$$
\frac{1 / \tau_{\mathrm{DP}}^{(1)}}{1 / \tau_{\mathrm{EY}}}=\frac{\alpha_{\mathrm{DP}}^{(1)}(t h) \tau_{p}^{2}(T)}{\alpha_{\mathrm{EY}}(t h) T}=\frac{7.5 \times 10^{15} \tau_{p}^{2}(T) \mathrm{K} \mathrm{ns}^{-2}}{T} .
$$

Even if the momentum relaxation time taken to be unrealistically low, say $1 \mathrm{fs}$, the DP mechanism is still nearly 2 orders of magnitude more efficient at relaxing spins than the EY mechanism in the temperature range studied here. Due to the drastic qualitative and quantitative differences between relaxation mechanisms, we have unequivocally determined the relevant conduction electron spin relaxation mechanism in $\mathrm{ZnO}$.

The fit of theory to the experimental data is clearly very good. We found that no reasonable fit was possible using only a single impurity level, though this worked very well for GaAs, ${ }^{6}$ so we used two levels. A good fit by this method was possible by adjusting the coefficients $\alpha_{\mathrm{DP}}^{(1)}(\exp )$ and $\alpha_{\mathrm{DM}}(\exp )$, and the binding energies $\varepsilon_{l s}, \varepsilon_{l d}$ and concentrations $n_{\mathrm{imp}, s}, n_{\mathrm{imp}, d}$ of the two donors, subject to the constraint that the room-temperature carrier density should equal the measured $^{4}$ value of $1.26 \times 10^{15} \mathrm{~cm}^{-3}$. Qualitatively, one finds that $n_{\mathrm{imp}, d} \gg n_{\mathrm{imp}, s}$ and $\left|\varepsilon_{l d}\right| \gg\left|\varepsilon_{l s}\right|$ to get the right order of magnitude of the relaxation at low $T$. Physically, the deep impurity spins are important because they relax the shallow impurity spins by the DM mechanism, and the strength of the low $T$ relaxation implies that the deep impurities must be quite numerous. Quantitatively, a least-squares fit to the data of Ref. 4 yields $\alpha_{\mathrm{DP}}^{(1)}(\exp )=134.5 \mathrm{~K}^{-1} \mathrm{~ns}^{-2}, \alpha_{\mathrm{DM}}(\exp ) n_{\mathrm{imp}, d}$ $=0.06 \mathrm{~ns}^{-1},\left|\varepsilon_{l d}\right|=360 \mathrm{meV},\left|\varepsilon_{l s}\right|=23 \mathrm{meV}$, and $n_{\mathrm{imp}, s}=6.0$ $\times 10^{14} \mathrm{~cm}^{-3}$.

$\alpha_{\mathrm{DP}}^{(1)}(\exp )$ is about four times larger than the theoretical value of $\alpha_{\mathrm{DP}}^{(1)}(t h)$ given above, possibly due to strain effects. We also note that the values of $\tau_{p}$ that we used were taken from a different sample.

If we take $n_{\text {imp }, d}$ to be near the highest values measured for the deep donor (see below) then $\alpha_{\mathrm{DM}}(\exp )=12$ $\times 10^{-20} \mathrm{~cm}^{3} \mathrm{~ns}^{-1}$ is about 1 order of magnitude larger than the theoretical estimate $\alpha_{\mathrm{DM}}(t h)$ given above. In view of the very poor understanding of the impurity wave functions, and the exponential dependence of $\alpha_{\mathrm{DM}}$ on the overlaps, this is perhaps not too disturbing.

The presence of a shallow donor and a very deep donor has been seen in hydrothermally grown $\mathrm{ZnO}$ samples of the type investigated here. ${ }^{9,31}$ Donor concentrations up to nearly $5.0 \times 10^{17} \mathrm{~cm}^{-3}\left(n_{\mathrm{imp}, d}\right)$ have been measured for donors 
330-360 meV $\left(\left|\varepsilon_{l d}\right|\right)$ deep. ${ }^{9,31,32}$ Donors as shallow as 13-51 meV $\left(\left|\varepsilon_{l s}\right|\right)$ have been measured ${ }^{8}$ at lower concentrations $\sim 5.0 \times 10^{14} \mathrm{~cm}^{-3}\left(n_{\mathrm{imp}, s}\right)$. Comparison with our values indicates that the parameters extracted from the fit are very reasonable for this material.

From this analysis, we predict that in $\mathrm{ZnO}$ samples with fewer deep impurities, the relaxation time at low temperatures can be increased. As the impurities of $\mathrm{ZnO}$ vary greatly between different growth techniques, ${ }^{33}$ this prediction could be tested by further optical orientation experiments on different samples.

\section{CONCLUSIONS}

We have found that $\tau_{s}$ in bulk $\mathrm{ZnO}$ can be understood by invoking previously known spin relaxation mechanisms. The dominant mechanisms in the material turn out to be the DP (scattering) relaxation of the conduction electron spins for $T>50 \mathrm{~K}$ and the DM (anisotropic exchange) mechanism for the localized spins for $T<50 \mathrm{~K}$. In addition, it is very important to include the cross-relaxation between localized and conduction states previously proposed for GaAs. These physical ingredients explain quantitatively the relatively fast relaxation at low temperatures as being due mainly to the DM mechanism which in turn depends on having both deep and shallow impurity states. At high temperatures, the conduction states are dominant, and the DP mechanism gives an excellent fit to the data. The combination explains the very surprising nonmonotonic temperature dependence of $\tau_{s}$.

Finally, there are two aspects of the data in Ref. 4 that we have not addressed here: the applied magnetic-field dependences on the spin relaxation and the spin relaxation observed in $\mathrm{ZnO}$ epilayers. We plan on addressing the former issue in a future publication. As for the latter issue, the epilayers are doped 3-4 orders of magnitude higher than in the bulk case. At such high dopings, spin glass effects become important and localized donor states coalesce to produce donor bands; we do not expect our theory to be applicable in such a regime. The theory has now been sufficiently developed that optical orientation experiments can actually serve as a characterization tool for doped semiconductors, giving information about the binding energies and concentrations of the electrically active impurities in $n$-type materials.

\section{ACKNOWLEDGMENTS}

We would like to acknowledge useful discussions with $\mathrm{S}$. Ghosh. Financial support was provided by the National Science Foundation, Grants No. NSF-ECS-0523918 (N.J.H. and W.O.P.) and No. NSF-ECS-0524253 (R.J.).
${ }^{1}$ Ü. Özgür, Ya. I. Alivov, C. Liu, A. Teke, M. A. Reshchikov, S. Doğan, V. Avrutin, S.-J. Cho, and H. Morkoç, J. Appl. Phys. 98, 041301 (2005).

${ }^{2}$ T. Dietl, H. Ohno, F. Matsukura, J. Cibert, and D. Ferrand, Science 287, 1019 (2000)

${ }^{3}$ W. J. Fan, J. B. Xia, P. A. Agus, S. T. Tan, S. F. Yu, and X. W. Sun, J. Appl. Phys. 99, 013702 (2006).

${ }^{4}$ S. Ghosh, V. Sih, W. H. Lau, and D. D. Awschalom, Appl. Phys. Lett. 86, 232507 (2005).

${ }^{5}$ J. M. Kikkawa and D. D. Awschalom, Phys. Rev. Lett. 80, 4313 (1998).

${ }^{6}$ W. O. Putikka and R. Joynt, Phys. Rev. B 70, 113201 (2004).

${ }^{7}$ M. I. D'yakonov and V. I. Perel', Sov. Phys. JETP 33, 1053 (1974).

${ }^{8}$ H. von Wenckstern, H. Schmidt, M. Grundmann, M. W. Allen, P. Miller, R. J. Reeves, and S. M. Durbin, Appl. Phys. Lett. 91, 022913 (2007).

${ }^{9}$ U. Grossner, S. Gabrielsen, T. M. Børseth, J. Grillenberger, A. Y. Kuznetsov, and B. G. Svensson, Appl. Phys. Lett. 85, 2259 (2004).

${ }^{10}$ L. C. Lew Yan Voon, M. Willatzen, M. Cardona, and N. E. Christensen, Phys. Rev. B 53, 10703 (1996).

${ }^{11}$ M. I. Dyakonov and V. I. Perel, in Optical Orientation, edited by F. Meier and B. P. Zachachrenya (North-Holland, Amsterdam, 1984).

${ }^{12}$ K. Seeger, Semiconductor Physics, 6th ed. (Springer, New York, 1997), p. 161.

${ }^{13}$ C. P. Slichter, Principles of Magnetic Resonance, 3rd ed. (Springer, New York, 1990), pp. 192-207.
${ }^{14}$ J. R. Schrieffer and P. A. Wolff, Phys. Rev. 149, 491 (1966).

${ }^{15}$ R. J. Elliott, Phys. Rev. 96, 266 (1954).

${ }^{16}$ J. N. Chazalviel, Phys. Rev. B 11, 1555 (1975).

${ }^{17}$ G. L. Bir, A. G. Aronov, and G. E. Pikus, Sov. Phys. JETP 42, 705 (1976).

${ }^{18}$ Pil Hun Song and K. W. Kim, Phys. Rev. B 66, 035207 (2002).

${ }^{19}$ I. Dzyaloshinsky, Phys. Chem. Solids 4, 241 (1958).

${ }^{20}$ T. Moriya, Phys. Rev. 120, 91 (1960).

${ }^{21}$ L. P. Gor'kov and P. L. Krotkov, Phys. Rev. B 67, 033203 (2003).

${ }^{22}$ K. V. Kavokin, Phys. Rev. B 69, 075302 (2004).

${ }^{23}$ I. Žutić, J. Fabian, and S. Das Sarma, Rev. Mod. Phys. 76, 323 (2004).

${ }^{24}$ G. Dresselhaus, Phys. Rev. 100, 580 (1955).

${ }^{25}$ E. I. Rashba, Sov. Phys. Solid State 2, 1109 (1960).

${ }^{26}$ J. Y. Fu and M. W. Wu, J. Appl. Phys. 104, 093712 (2008).

${ }^{27}$ W. Weber, S. D. Ganichev, S. N. Danilov, D. Weiss, and W. Prettl, Appl. Phys. Lett. 87, 262106 (2005).

${ }^{28}$ W.-T. Wang, C. L. Wu, S. F. Tsay, M. H. Gau, Ikai Lo, and H. F. Kao, Appl. Phys. Lett. 91, 082110 (2007).

${ }^{29}$ G. E. Pikus and A. M. Titkov, in Optical Orientation, edited by F. Meier and B. P. Zachachrenya (North-Holland, Amsterdam, 1984).

${ }^{30}$ D. C. Look, Surf. Sci. 601, 5315 (2007).

${ }^{31}$ C. H. Seager and S. M. Myers, J. Appl. Phys. 94, 2888 (2003).

${ }^{32}$ G. H. Kassier, M. Hayes, and F. D. Auret, J. Appl. Phys. 102, 014903 (2007).

${ }^{33}$ D. C. Look, Mater. Sci. Eng., B 80, 383 (2001). 